Andreas Gruner · Thomas J. Hockertz · Heinrich Reilmann

Unfallchirurgische Klinik, Städtisches Klinikum Braunschweig

\title{
Alternative Verfahren bei distalen Femurfrakturen
} Minimalinvasive Plattenosteosynthese

\section{Zusammenfassung}

In der Versorgung der distalen Femurfraktur ist die Plattenosteosynthese ein etabliertes Verfahren. Das Less-Invasive-StabilisationSystem (LISS DF) als anatomisch vorgeformter und perkutan eingebrachter Fixateur interne am distalen Femur wird mit monokortikalen, winkelstabilen Schrauben verankert. Die anterolaterale Arthrotomie erlaubt die Gelenkrekonstruktion. Das Verfahren wird mit klinische Beispielen und Studienergebnissen dargestellt.

\section{Schlüsselwörter}

Distale Femurfraktur · Less-Invasive-Stabilisation-System (LISS) · Fixateur interne · Minimalinvasive Therapie ie operative Versorgung distaler Femurfrakturen unterliegt einem kontinuierlichen Wandel. Im Gegensatz zu vormals durchgeführten offenen Repositionstechniken mit Frakturfreilegung und Weichteildenudierung, verbunden mit einer hohen Rate primärer und sekundärer Spongiosaplastiken [12], kommen heute geschlossene Repositionstechniken, neue Implantattechnologien und Implantatplatzierungstechniken zum Einsatz. Diese setzen neue Erkenntnisse der minimalinvasiven Osteosynthese, Implantatfunktionalität und Knochenheilung konsequent um [2].

Ziel der Behandlung distaler Femurfrakturen muss die korrekte Wiederherstellung der Gelenkkongruenz verbunden mit korrekten Achsenverhältnissen und primärer Übungs- und Belastungsstabilität sein. Eine frühfunktionelle Nachbehandlung sollte gewährleistet sein.

\section{Instrumentarium und Implantate}

Insgesamt stehen für die Behandlung distaler Femurfrakturen unterschiedliche Osteosyntheseverfahren und Implantate zur Verfügung. Dazu zählen die gedeckte, BV-gesteuerte Osteosynthese, die offene Rekonstruktion durch Platten- und Schraubenosteosynthese (Kondylenabstützplatte, DCS) oder die Therapie mittels Fixateur externe (monolateral, Hybridfixateur, Ringfixateur nach Ilizarov mit/ohne Verfahrenswechsel). In letzter Zeit finden verstärkt intramedulläre Kraftträger (retrograder Marknagel,
DFN [4]) Verwendung. Neu entwickelte, winkelstabile Implantate wie das hier näher beschriebene LISS DF stabilisieren die Fraktur im Sinn eines Fixateur interne und zeigen neue Lösungsmöglichkeiten bei Implantatverankerungsproblemen z. B. bei osteoporotischem Knochen oder periprothetischen Frakturen.

\section{Vergleich der Osteosyntheseverfahren}

Im Vergleich der Osteosyntheseverfahren bezogen auf Deformation und Biegungssteifigkeit am distalen Femur wurde das LISS gegen die Kondylenabstützplatte und die DCS experimentell untersucht. Dabei zeigte das LISS

- eine geringere irreversible Deformation,

- eine höhere elastische Deformation und

- eine insgesamt geringere Biegungssteifigkeit des Systems [11].

Diese Ergebnisse weisen darauf hin, dass das LISS eine verbesserte Fähigkeit besitzt, größeren Lasten standzuhalten. Grundlage hierzu sind monokortikal eingebrachte und winkelstabil verankerte Schrauben. Die höhere elastische

Dr. Andreas Gruner Unfallchirurgische Klinik, Städtisches Klinikum Braunschweig, Holwedestraße 16,38118 Braunschweig, E-Mail:Andreas_Gruner@gmx.de, Tel.:0531-5951257, Fax:0531-5951462 


\section{A. Gruner · T.J. Hockertz $\cdot$ H. Reilmann}

Alternative techniques for use in distal fractures of the femur Minimally invasive osteosynthesis with plates

\section{Abstract}

Plate osteosynthesis is a well-established procedure in the treatment of distal femoral fracture. The Less Invasive Stabilisation System (LISS DF) is a percutaneously placed interlocking internal fixator with angular stability. The plates are pre-shaped. For intra-articular fractures a anterolateral arthrotomy is recommended. This procedure is presented with clinical examples and study results.

\section{Keywords}

Distal femoral fracture - Less Invasive Stabilisation System (LISS) · Internal fixator · Minimally invasive therapy
Abb. 2 a , $b>$ Zugänge: a laterale Hautinzision proximal des TubercuIum Gerdyi für A-Frakturen, b anterolaterale parapatellare Arthrotomie bei Gelenkbeteiligung (C-Frakturen)
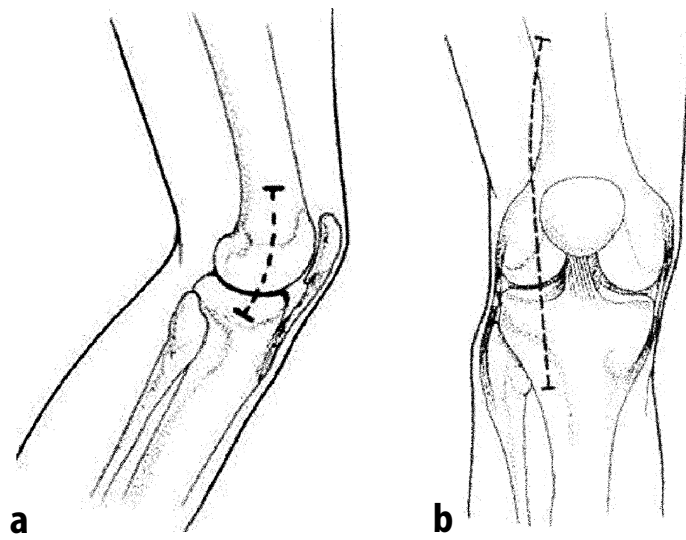

Deformation des LISS kann durch eine geringere Biegungssteifigkeit aufgrund der Materialwahl erklärt werden.

\section{LISS-Implantatbeschreibung}

Bei dem LISS DF (Less-Invasive-Stabilisation-System) für das distale Femur handelt es sich um einen extramedullären Fixateur interne. Der anatomisch vorgeformte Kraftträger zeichnet sich durch eine minimalinvasive Insertion bzw. Platzierung aus. Dabei wird auf eine Freilegung der Frakturzone verzichtet, und die Knochenfragmente werden im Weichteilverbund belassen [3]. Im Rahmen einer biologischen Osteosynthesetechnik (MIPPO) wird die periostale Perfusion geschützt, femorale Versorgungsarterien und Perforansgefäße werden erhalten [1]. Die Fixation der winkelstabil im Implantat verankerten selbstschneidenden und selbstbohrenden Schrauben erfolgt monokortikal.
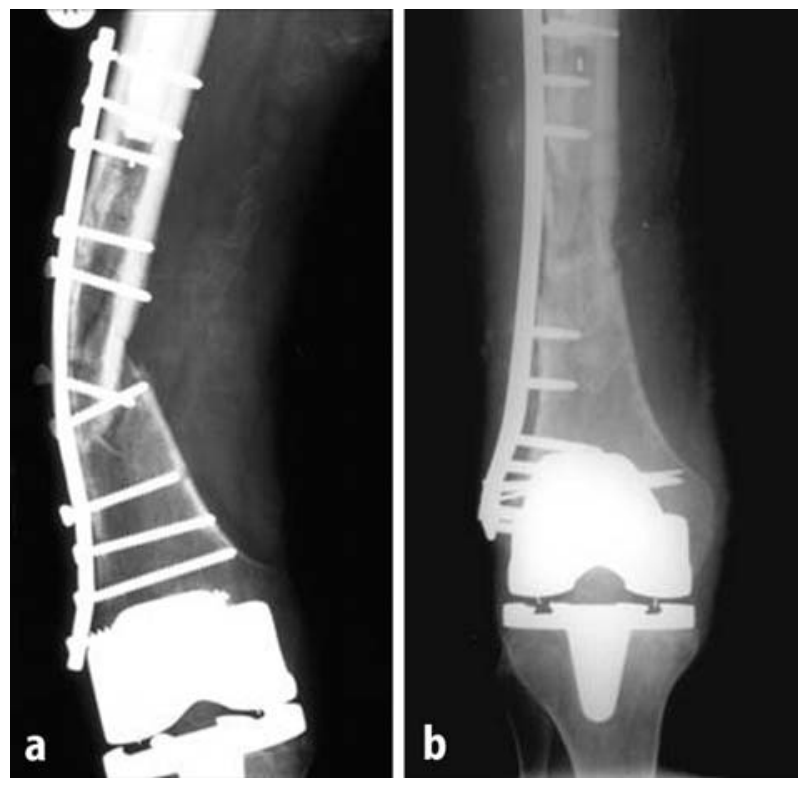

Abb. $1 a, b<$ Plattendeformation bei interprothetischer Femurfraktur und Reosteosynthese mit LISS DF

\section{Indikation für LISS DF}

Die Indikation für das LISS DF besteht für Frakturen der AO-Klassifikation 33A1-A 3 und 33 $\mathrm{C}_{1}-\mathrm{C}_{3}$. Die Anwendungsgebiete beinhalten geschlossene und offene Frakturen, peri- und interprothetische Frakturen [5] sowie Frakturen distal eines proximal liegenden Implantats (z. B. PFN, $\gamma$-Nagel, DHS) (Abb. 1).

\section{Operationstechnik}

Die Längenauswahl des LISS erfolgt im Rahmen der Frakturklassifikation, die am Insertionsbügel zu besetzenden Schraubenlöcher können danach geplant werden.

Das zu operierende Bein sollte frei beweglich mit einem in $30^{\circ}$ Flexion gelagerten Kniegelenk abgedeckt werden [10]. Der Patient wird auf einem röntgendurchlässigen Standardtisch gelagert.

Als Zugangsweg werden die laterale Hautinzision proximal des Tuberculum Gerdyi für A-Frakturen oder eine anterolaterale parapatellare Arthrotomie bei Gelenkbeteiligung (C-Frakturen) empfohlen (Abb. 2) [8].

Der Tractus iliotibialis wird gespalten und das LISS zwischen M. vastus lateralis und Periost eingeführt. Der Zielbügel wird verschlossen. Es erfolgen die Reposition und die temporäre Fixation des LISS mittels Kirschner-Draht und Repositionsinstrumentarium am Knochen (Abb. 3).

Die Platzierung der Schaftschrauben und die Länge der Kondylenschrauben können präoperativ mittels einer Skizze und Schablone geplant werden. Die Schraubenplatzierung erfolgt über Stichinzision. Dabei sollten mindestens 3 monokortikale Schrauben in jedem Hauptfragment platziert werden. 


\section{Problemfrakturen}

Bei Frakturen mit Gelenkbeteiligung erfolgt primär die stufenlose Rekonstruktion der Gelenkflächen. Der
Gelenkblock wird unter Ausrichtung der Knochenlänge, -achse und -rotation mit der Diaphyse verbunden [9].

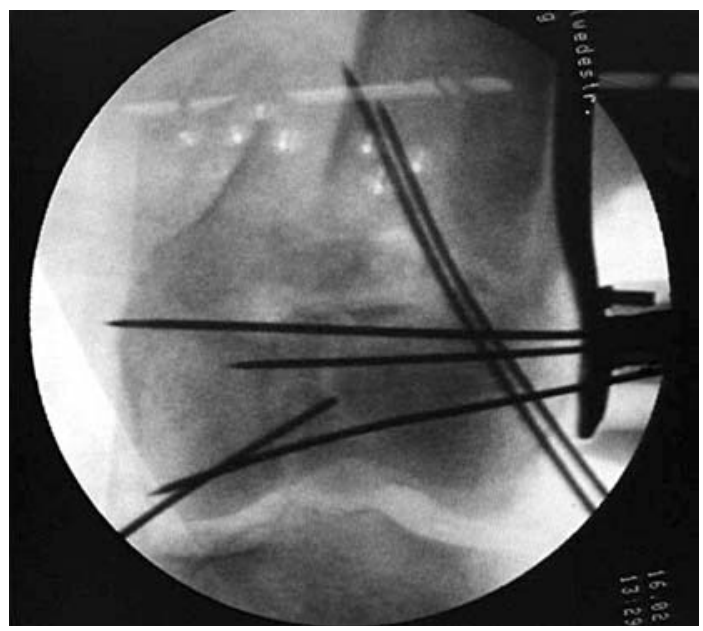

Abb. $3<$ Temporäre Fixation des LISS DF mittels Kirschner-Draht und Repositionsinstrumentarium am Knochen

Abb. 4 a-g $\nabla$ 28-jähriger Patient, A0-33C2-Fraktur (a, b), Primärversorgung (c, d,e), Frühmobilisation ab dem 2. Tag postoperativ, sichtbare Kallusformation ab der 4 . Woche postoperativ $(f, g)$

\section{Operative Therapie - Fallbeispiele}

\section{Fall 1}

In Abb. 4 ist der Fall eines 28-jährigen Patienten mit AO-33C2-Fraktur dargestellt.

\section{Fall 2}

In Abb. 5 ist der Fall einer 82-jährigen Patientin mit $\mathrm{AO}-33 \mathrm{C} 2$-Fraktur dargestellt.

\section{Fall 3}

In Abb. 6 ist der Fall einer 72-jährigen Patientin mit AO-33A2-Fraktur dargestellt.

\section{Ergebnisse}

\section{Eigene Anwendung LISS DF [6]}

Im eigenen Patientengut wurden in den letzten 24 Monaten 80 Femurfrakturen mit
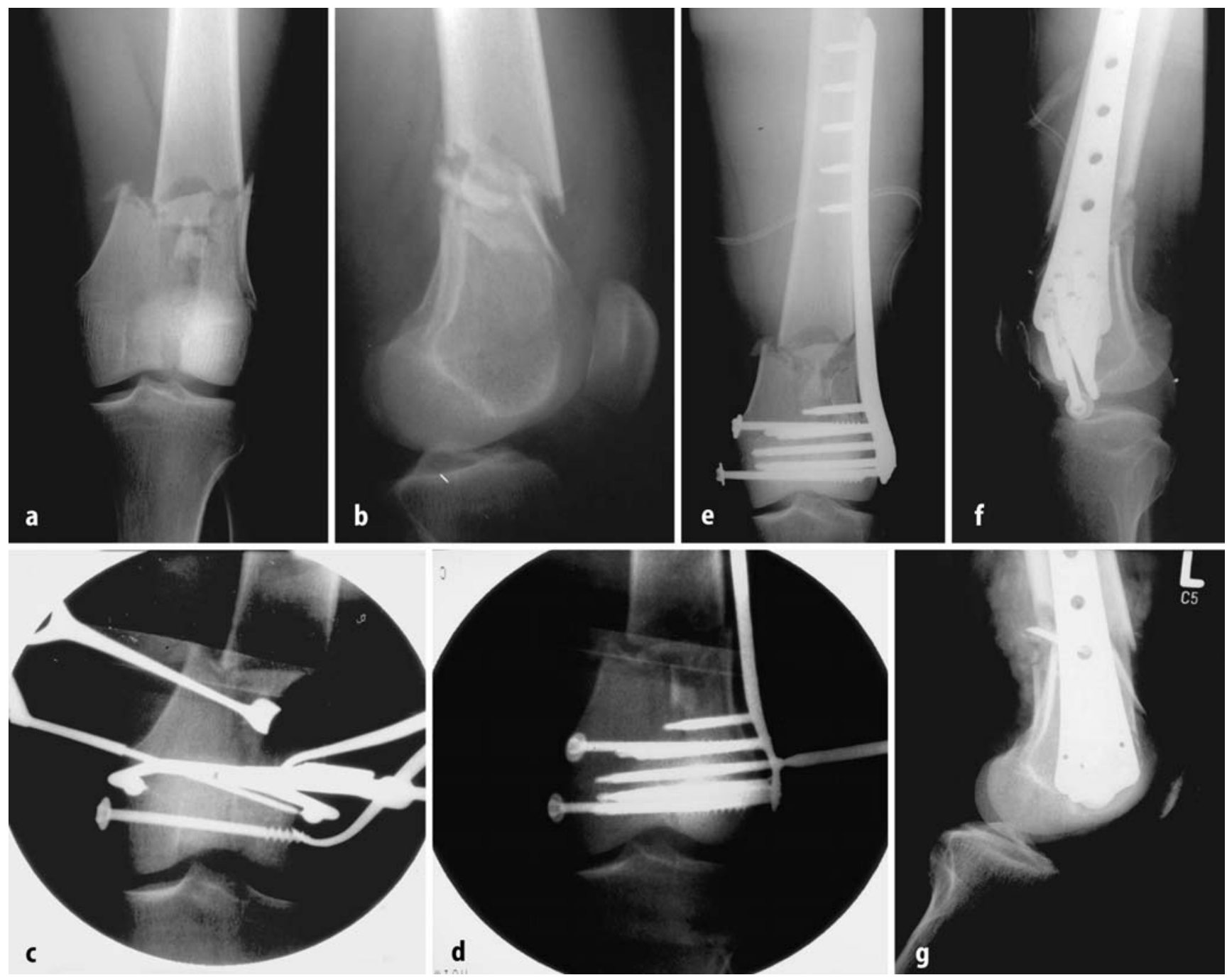

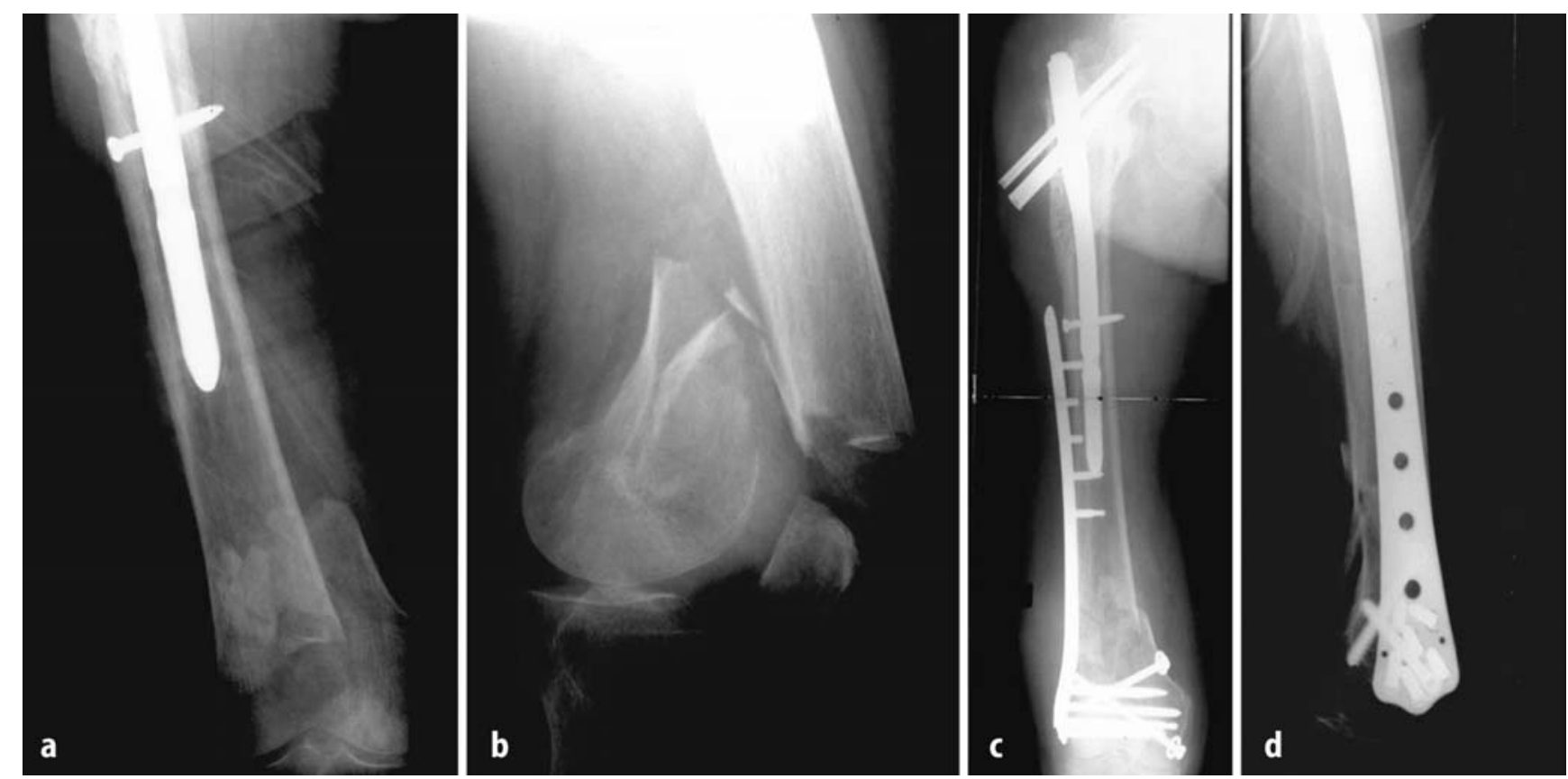

Abb. 5 a-d $\Delta$ 82-jährige Patientin, A0-33C2-Fraktur, liegender PFN (a, b), Primärversorgung, Frühmobilisation ab 2. Tag postoperativ ( $c, d)$

dem LISS DF versorgt. Die Frakturklassifikationen sind in Tabelle 1 dargestellt.

Alle 33 Patienten mit periprothetischer Fraktur konnten nachuntersucht werden. Dabei wurden 1 Plattenausriss und 1 Plattenbruch beobachtet. Infektionen und Frakturheilungsstörungen traten nicht auf.

\section{Prospektive Multizenterstudie LISS DF $(n=116)$ [15]}

Die Ergebnisse der prospektiven Multizenterstudie zu LISS DF von $n=116 \mathrm{~Pa}$ tienten sind in Tabelle 2 dargestellt [15].

In älteren Studien zur operativen Versorgung distaler Femurfrakturen wurden demgegenüber Infektionsraten von $22,8 \%$ und verzögerte Frakturhei-

Abb. 6 a-d $\nabla$ 72-jährige Patientin, A0-33A2-Fraktur, liegende Knieprothese (a, b), Primärversorgung, Frühmobilisation ab 2. Tag postoperativ $(c, d)$
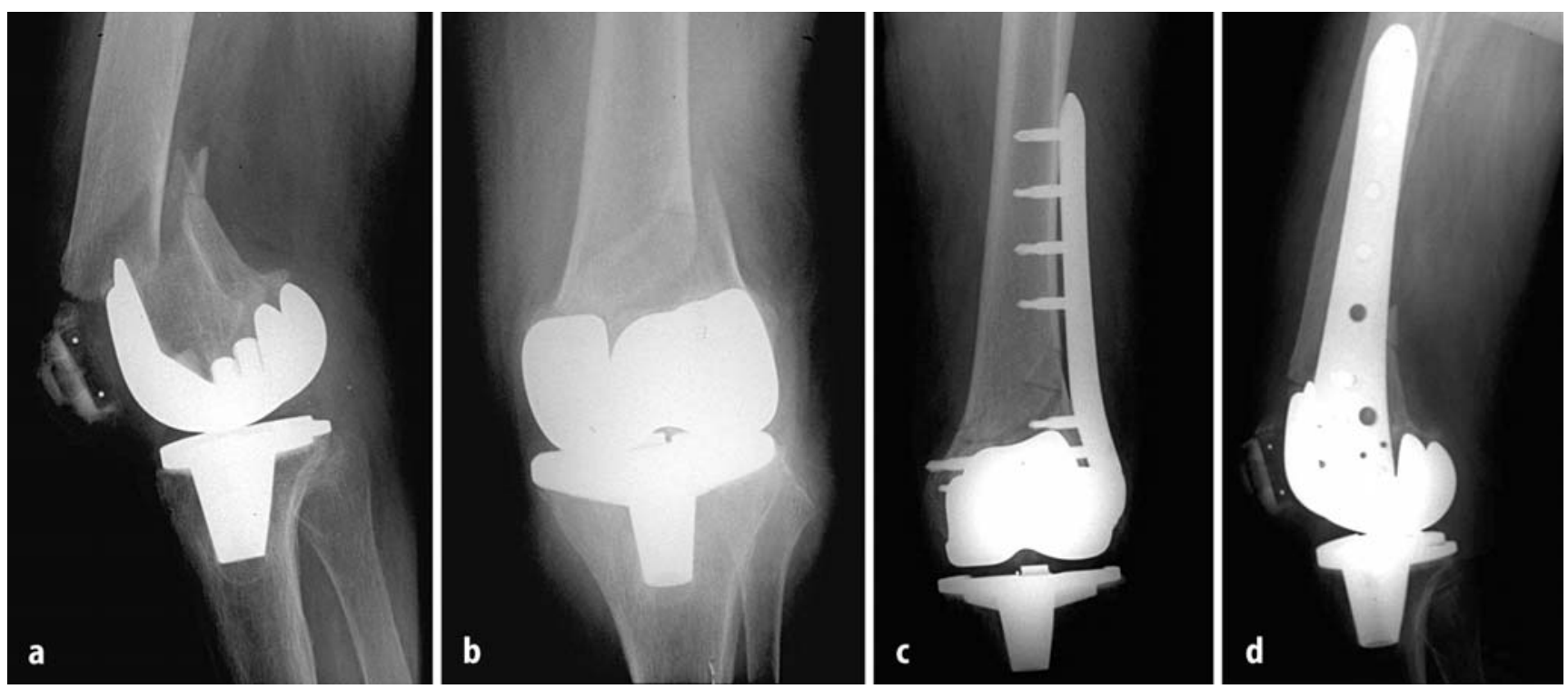
Tabelle 1

Frakturklassifikationen im eigenen Patientengut bei Versorgung durch LISS DF

Fraktur Anzahl (gesamt: $n=80$ )

A0 32A-C 13

$\mathrm{A} 033 \mathrm{~A}+\mathrm{C} \quad 28$

Johannson II+III 23

Liegende Knieprothese 5

Interprothetisch 5

Sonderindikationen 6

wurden bei technisch bedingter Fehlplatzierung, Osteoporose und Fehlbelastung beobachtet.

Eine Restitutio ad integrum ist auch mit neuen Operationstechniken in Anbetracht der Schwere der Verletzung (AOC-Fraktur) nur selten zu erreichen [14].

Tabelle 2

Ergebnisse der prospektiven Multizenterstudie LISS DF

Komplikation

Anteil

(gesamt:

$n=116$ )

[\%]

\section{Infektion}

2,6

Verzögerte Frakturheilung

Implantatlockerung

4,3

Implantatbruch

4,3

Varus-/Valgusfehlstellung $>10^{\circ} \quad 1,7$

Ante-/Rekurvation $>10^{\circ} \quad 2,6$

Heterotope Ossifikation [13] $\quad 3,4$

Thrombose [13]

\section{Resümee}

Die Vorteile bei der Verwendung des LISS DF bei der Versorgung der distalen Femurfraktur liegen in einem minimalinvasiven Osteosyntheseverfahren, welches die Grundlagen der biologischen Osteosynthese berücksichtigt. Begleitende Gelenkbeteiligungen können über differenzierte Zugangswege parallel versorgt werden. Das Verfahren eignet sich insbesondere zur Versorgung inter- und periprothetischer Frakturen am Femur sowie auch bei Femurfrakturen und liegendem proximalem Implantat. Es ist bei schwerem Weichteilschaden anwendbar. Eine Spongiosaplastik ist nur in Ausnahmefällen notwendig.

Die Schwierigkeiten liegen in der genauen Wiederherstellung der Achsverhältnisse.

Es handelt sich um ein kostenintensives Implantat, das eine exakte Operationstechnik erfordert.

In den bisher veröffentlichten Studien der klinischen Anwendung wurden bei breitem Indikationsspektrum des Less-Invasive-Stabilisation-System LISS DF - für die distale Femurfraktur gute Ergebnisse erzielt.

\section{Literatur}

1. Farouk 0 , Krettek C, Miclau T, Schandelmaier P, Tscherne H (1998) Effects of percutaneous and conventional plating techniques on the blood supply to the femur. Arch Orthop Trauma Surg 117:438-441

2. Gautier E, Perren SM (1992) Die "Limited Contact Dynamic Compression Plate (LCDCP)" Biomechanische Forschung als Grundlage des neuen Plattendesigns. Orthopäde 21: 11-23

3. Gerber C, Mast J, Ganz R (1990) Biological internal fixation of fractures. Arch Orthop Trauma Surg 109:295-303
4. Grass R, Zwipp H (1998) Minimal-invasive Methode zur Behandlung von supra-diakondylären Femurfrakturen.ZentralbI Chir 123: 1247-1251

5. Hockertz TJ, Gruner A, Reilmann H (1999) Die Versorgung von periprothetischen Femurfrakturen bei liegender Knieprothese mit dem LISSystem. Ein neuer Therapieansatz. Unfallchirurg 102:811-814

6. Hockertz T, Gruner A, Reilmann H (2001) Versorgung peri- und interprothetischer Oberschenkelfrakturen mit dem LISS DF. Hefte Unfallchirurg 283:360-361

7. Koval KJ, Hoehl JJ, Kummer FJ et al. (1997) Distal femoral fixation: a biomechanical comparison of the standard condylar buttress plate, a locked buttress plate and the 95 -degree blade plate.J Orthop Trauma 11:521-524

8. Krettek C, Schandelmaier P, Tscherne H (1996) Distale Femurfrakturen: transartikuläre Rekonstruktion, perkutane Plattenosteosynthese und retrograde Nagelung. Unfallchirurg 99:2-10

9. Krettek C,Miclau T, Grün 0, Schandelmaier $P$, Tscherne $H$ (1998) Intraoperative control of axes, rotation and length in femoral and tibial fractures - technical note. Injury [Suppl] 29: 29-39

10. Krettek C, Schandelmaier P, Richter M, Tscherne H (1998) Distale Femurfrakturen. Swiss Surg 4: 263-278

11. Marti A, Fankhauser C, Frenk A, Cordey J, Gasser $B$ (2001) Biomechanical evaluation of the less invasive stabilization system for the internal fixation of the distal femur fractures.J Orthop Trauma 15:482-487

12. Neer CS, Grantham SA, Shelton ML (1967) Supracondylar fracture of the adult femur. J Bone Joint Surg Am 49-A:591-613

13. Schandelmaier P, Stephan C, Reimers N, Krettek C (1999) LISS-Osteosynthese von distalen Femurfrakturen. Trauma Berufskrankh 4:392-397

14. Schandelmaier P, Stephan C, Krettek C, Tscherne H (2000) Distale Femurfrakturen. Unfallchirurg 70:423-436

15. Schütz M, Müller M, Kääb M, Voigt C, Regazzoni P, Holzach P, Schandelmaier P, Höntzsch D, Werken C van der, Südkamp NP, Haas NP (2000) Minimalinvasive Frakturstabilisierung von distalen Femurfrakturen mit dem LIS-System Ergebnisse einer prospektiven MultizenterStudie. Acta Chir Austriaca [Suppl 161] 32:83-84

\section{Behandllungsstrategie bei Unterschenkelfrakturen mit Weichteilschaden}

